ORIGINAL ARTICLE

\title{
The p53-Mdm2 association in epithelial cells in idiopathic pulmonary fibrosis and non-specific interstitial pneumonia
}

\author{
N Nakashima, K Kuwano, T Maeyama, N Hagimoto, M Yoshimi, N Hamada, M Yamada, \\ Y Nakanishi
}

See end of article for authors' affiliations

Correspondence to: Dr K Kuwano, Research Institute for Diseases of the Chest, Graduate School of Medical Sciences, Kyushu University, 3-1-1, Maidashi, Higashi-ku, Fukuoka 812-8582, Japan; kkuwano@kokyu. med.kyushu-u.ac.jp

Accepted for publication 9 November 2004
Background: Wild-type p53 is increased during cellular responses to various stresses. Mdm2, which is induced by p53, regulates p53 protein concentrations through the ubiquitin-proteasome pathway.

Aim: To investigate whether the Mdm2 mediated ubiquitination of p53 is associated with epithelial cell apoptosis in idiopathic pulmonary fibrosis (IPF).

Methods: Immunohistochemistry and western blot analysis were carried out on lung samples obtained by lung biopsy from patients with IPF and non-specific interstitial pneumonia (NSIP).

Results: The expression of p53, phosphorylated p53, Mdm2, p21, and Bax was upregulated in epithelial cells from patients with IPF and NSIP compared with normal lung parenchyma. Except for p21, there was a significant increase in the expression of these factors in IPF compared with NSIP. In addition, the number of apoptotic cells and the number of p53 and Bax positive cells was increased compared with controls. p53 conjugated with Mdm2 was decreased in IPF compared with NSIP and controls. Ubiquitinated p53 was increased in both IPF and NSIP compared with controls.

Conclusions: Signalling molecules associated with p53 mediated apoptosis may participate in epithelial cell apoptosis, and the attenuation of p53-Mdm2 conjugation and of p53 degradation may be involved in the epithelial cell apoptosis seen in IPF. Augmented epithelial apoptosis in IPF may lead to the poor prognosis compared with NSIP.
$\mathrm{T}$ he ubiquitin-proteasome pathway is the major protein degradation pathway involved in proliferation, apoptosis, and homeostasis. This pathway degrades intracellular proteins by two steps. The first step comprises the attachment of multiple ubiquitin molecules to the target protein, and the second comprises the degradation of the targeted protein by proteasome. The attachment of ubiquitin is mediated by three enzymatic reactions, comprising El activating enzyme, E2 conjugating enzyme, and E3 ligases. Recognition of substrates by E3 ligases determines the specificity of the target proteins. Once ubiquitinated, the target proteins are rapidly degraded by $26 \mathrm{~S}$ proteasome. $^{12}$

Wild-type p53 normally acts to suppress cell growth while the cell attempts to repair DNA damage. It also promotes apoptosis in those cells that have irreparably damaged DNA or that continue to proliferate. ${ }^{3}{ }^{4}$ Expression of wild-type p53 is very low in normal cells, but is greatly upregulated in response to a variety of stresses. ${ }^{5-7}$ The expression and function of the p53 protein is regulated mainly at the posttranscriptional level.

\section{"Mdm2 and p53 form an autoregulatory feedback loop"}

$\mathrm{Mdm} 2$ is the most important cellular regulator of p53. Mdm2 can function as an E3 ubiquitin ligase, ${ }^{8}$ mediate the ubiquitination of nuclear and cytoplasmic p53, ${ }^{9}$ and induce the degradation of $\mathrm{p} 53$ protein. ${ }^{10}{ }^{11} \mathrm{Mdm} 2$ inhibits the apoptotic function of p53 mainly by targeting it for degradation. ${ }^{12}$ Upregulation of $\mathrm{p} 53$ induces the transcription of $\mathrm{Mdm} 2$, whereas $\mathrm{Mdm} 2$ protein binds to $\mathrm{p} 53$ and inhibits its transcriptional activity. ${ }^{13}{ }^{14}$ Accordingly, Mdm2 and p53 form an autoregulatory feedback loop. ${ }^{15}$ However, phosphorylation of p53 at Ser15 attenuates both the p53-Mdm2 interaction ${ }^{16}$ and $\mathrm{p} 53$ nuclear export. ${ }^{17}$
The mitochondria mediated apoptosis pathway is crucial for p53 mediated apoptosis, and involves Bcl-2 family proteins. p53 mediates apoptosis by transcriptional activation of proapoptotic genes, such as PUMA, Bax, Fas, and Apaf- $1 .{ }^{18} 19$ Epithelial cell apoptosis can be identified in diffuse alveolar damage and is associated with p53 and Bax expression. $^{20} 21$

p21 (Wafl/Cipl/Sdil) is induced in cells that contain wildtype p53 after cellular stress, and is a crucial downstream effector in the p53 specific pathway of growth control in mammalian cells. ${ }^{22}$ p21 enhances survival either by promoting DNA repair or by modifying cell death caused by exposure to hyperoxia, radiation, or cytotoxic agents. ${ }^{23-25}$ These findings suggest that $\mathrm{p} 2 \mathrm{l}$ may be a key regulator of DNA replication and repair after lung injury.

We previously demonstrated that DNA damage and apoptosis are associated with the upregulation of p53 and p21 protein in bronchiolar and alveolar epithelial cells in IPF. ${ }^{26}$ We also showed that the number of apoptotic epithelial cells was significantly increased in IPF and NSIP compared with normal lung parenchyma, and was significantly correlated with cytochrome c release from the mitochondria and with the expression of cleaved caspase 3 in epithelial cells. ${ }^{27}$ As an extension of our previous study, we investigated the interaction of p53 and Mdm2, and the post-translational modification of p53 involving phosphorylation and ubiquitination in IPF and NSIP. In addition, we investigated the association between the modification of p53 and the apoptotic response involving Bax versus p2l expression in lung epithelial cells of IPF and NSIP.

Abbreviations: IPF, idiopathic pulmonary fibrosis; JNK, c-jun $\mathrm{N}$ terminal kinase; NSIP, non-specific interstitial pneumonia; TUNEL, terminal deoxynucleotidyl transferase mediated dUTP nick end labelling 


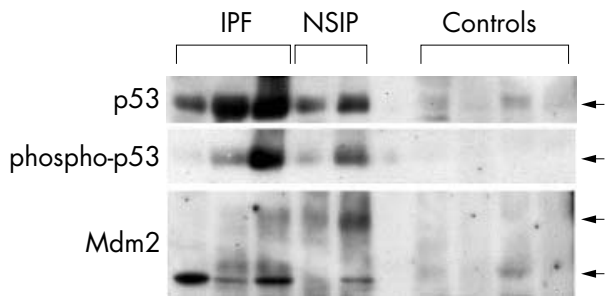

Figure 1 Representative results of western blot analysis for p53, phosphorylated p53 (phospho-p53), and Mdm2 proteins in lung tissue from patients with idiopathic pulmonary fibrosis (IPF) and non-specific interstitial pneumonia (NSIP) and for normal lung parenchyma (controls). In the panel showing Mdm2, the upper and lower arrows indicate the active $(90 \mathrm{kDa})$ and inactive $(60 \mathrm{kDa})$ forms, and the optical density of the upper bands was used for quantitative analysis.

\section{MATERIALS AND METHODS}

Our study was performed on 13 lung samples obtained by thoracoscopic lung biopsy. The diagnosis of IPF and NSIP was established by a combination of medical history, physical examination, laboratory tests, chest roentgenograms, pulmonary function tests, and the results of histological findings, according to previously described criteria. ${ }^{28}$ The histological findings of lung biopsy specimens were compatible with those of IPF in eight patients and NSIP in five. All eight patients with IPF were men, whose ages ranged from 42 to 67 years (mean, 58.6). Seven were smokers and one was a non-smoker. The patients with NSIP comprised three men and two women, whose ages ranged from 50 to 68 years (mean, 58.2). Two were smokers and three were nonsmokers. The results from the IPF and NSIP specimens were compared with those from eight normal lung parenchyma specimens obtained by lobectomy for lung cancer of a solitary pulmonary nodule. The patients with lung cancer comprised five men and three women, whose ages ranged from 56 to 78 years (mean, 67), and all were smokers. After the lung tissue was obtained, it was immediately frozen in liquid nitrogen and stored at $-80^{\circ} \mathrm{C}$ for western blot analysis. Formalin fixed, paraffin wax embedded lung tissues were used for terminal deoxynucleotidyl transferase mediated dUTP nick end labelling (TUNEL) staining and immunohistochemistry.

The specific antibodies used in our study were as follows: mouse monoclonal anti-p53 antibody (Ab-2; Oncogene Research Products, Darmstadt, Germany), rabbit antiphospho-p53 (Ser15) antibody (Cell Signaling Technology, Beverly, Massachusetts, USA), mouse monoclonal antiMdm2 antibody (Ab-1; Oncogene Research Products), mouse anti-ubiquitin monoclonal antibody (Santa Cruz Biotechnology, Santa Cruz, California, USA), mouse antimulti-ubiquitin monoclonal antibody (Medical and Biological Laboratories, Nagoya, Japan), rabbit anti-Bax polyclonal antibody (Oncogene Research Products), and mouse anti-p21 monoclonal antibody (Oncogene Research Products). The same antibodies were used for western blot analysis and immunohistochemistry.

\section{Immunoblots and immunoprecipitation}

Frozen lung tissues were homogenised in buffer A $(25 \mathrm{mM}$ Hepes (pH 7.5), 5mM $\mathrm{MgCl}_{2}$, ImM EGTA, ImM phenylmethyl sulfonyl fluoride, $1 \mu \mathrm{g} / \mathrm{ml}$ leupeptin, and $1 \mu \mathrm{g} / \mathrm{ml}$ aprotinin) using a polytron homogeniser (Kinematica, Luzern, Switzerland). The supernatant was dissolved in sample buffer (133mM Tris $\mathrm{HCl}(\mathrm{pH} \mathrm{6.8)}, 0.1 \%$ sodium dodecyl sulfate, $5 \%$ glycerol, $0.67 \%$ 2-mercaptoethanol, $1 \mu \mathrm{g} / \mathrm{ml}$ leupeptin, and $1 \mu \mathrm{g} / \mathrm{ml}$ aprotinin) and boiled. The homogenate was centrifuged at $15000 \times g$ for 30 minutes at $4^{\circ} \mathrm{C}$. Immunoprecipitation was performed under
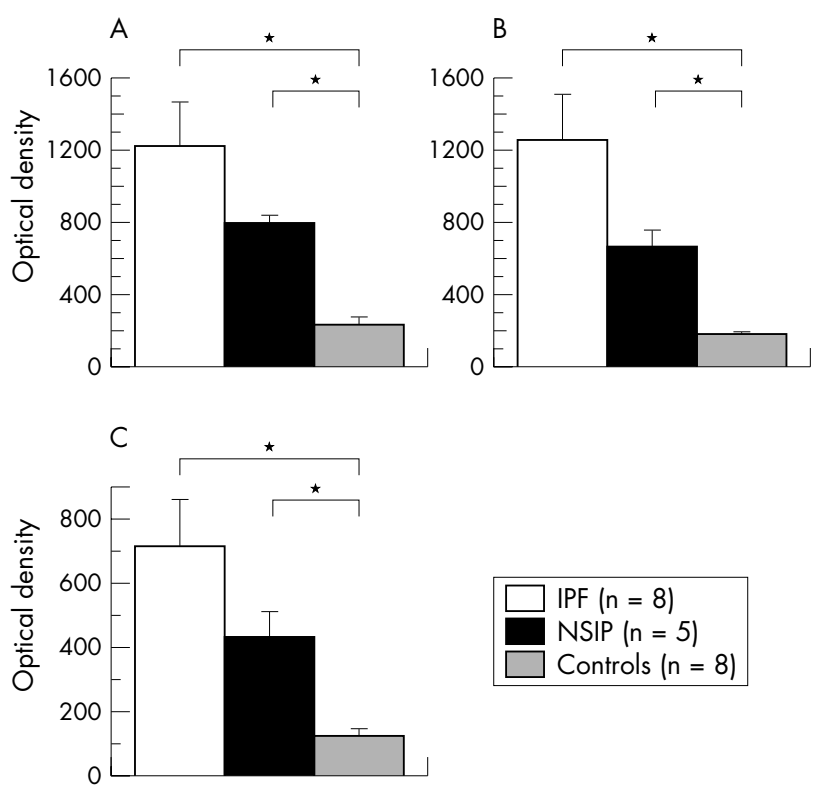

Figure 2 Quantitative results of western blot analysis for (A) p53, (B) phosphorylated p53 (at Ser 15), and (C) Mdm2 proteins in lung tissue from patients with idiopathic pulmonary fibrosis (IPF; $n=8)$, non-specific interstitial pneumonia (NSIP; $n=5)$, and controls $(n=8)$. ${ }^{*} p<0.01$.

non-reducing conditions on a rotator for three hours at $4{ }^{\circ} \mathrm{C}$ with $20 \mu \mathrm{l}$ of protein G Sepharose (Amersham, Uppsala, Sweden) and $2 \mu \mathrm{g}$ of anti-p53 antibody. Protein concentrations were determined using the Bio-Rad protein assay (BioRad Laboratories, Hercules, California, USA). Aliquots of $30 \mu \mathrm{g}$ of protein were loaded on to each column. The proteins were separated by sodium dodecyl sulfate polyacrylamide gel electrophoresis. After electrophoresis, the proteins were transferred on to a polyvinylidene fluoride hydrophobic membrane (Millipore, Bedford, Massachusetts, USA). The membranes were incubated with specific antibodies in blocking buffer at $4^{\circ} \mathrm{C}$ overnight. The blots were developed using an ECL western blotting detection kit (Amersham Pharmacia Biotech, Little Chalfont, Buckinghamshire, UK), as described previously. ${ }^{27}$ Pictures of the membranes were taken and scanned. The images were analysed using NIH image Ver.1.61 (National Institutes of Health, Washington, USA). The sum of the optical density of several bands in the ubiquitinated p53 protein was calculated to analyse the statistical differences.

\section{Immunohistochemistry}

After dewaxing in xylene and rehydration in ethanol, $5 \mu \mathrm{m}$ thick paraffin wax embedded sections were autoclaved at $120^{\circ} \mathrm{C}$ for five minutes in a glass pot filled with enough distilled water to immerse the sections completely, with the exception of those sections intended for immunohistochemical analysis of Bax and multi-ubiquitin. Immunohistochemistry was performed using a modified streptavidin-biotinylated peroxidase technique using a Histofine SAB-PO kit from Nichirei Corporation (Tokyo, Japan), as described previously. ${ }^{27}$ The sections were counterstained with methyl green and mounted. The number of positive cells/200 alveolar epithelial cells was counted in 20 randomly selected fields for each section at $\times 200$ magnification, and shown as the percentage of epithelial cells.

For each section, the grade of fibrosis in 20 randomly selected fields at $\times 200$ magnification was also assessed at the same time as the sections were graded for 

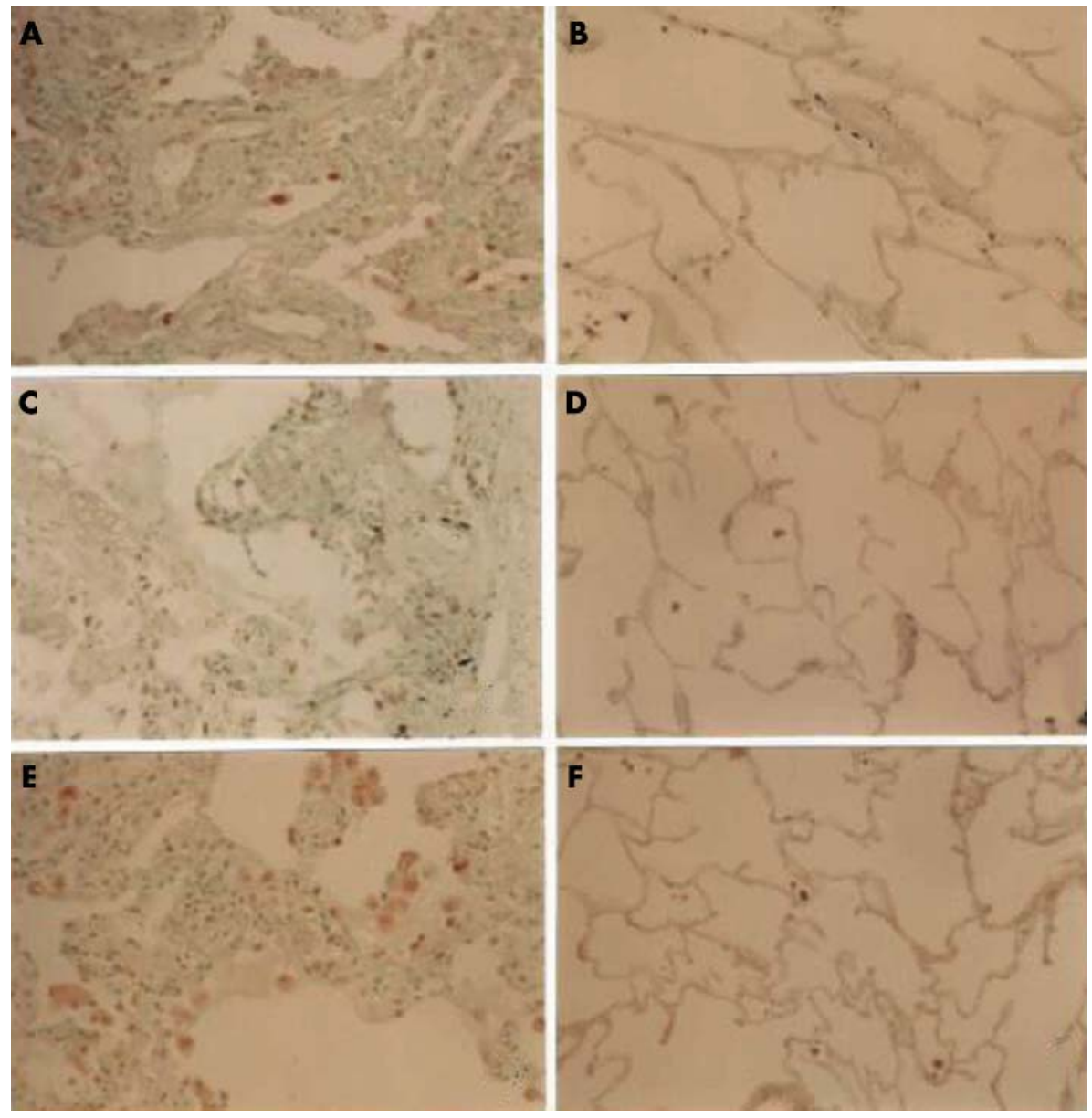

Figure 3 Representative results of immunohistochemical analysis for p53, phosphorylated p53 (at Ser 15), and Mdm2 proteins in lung tissue from patients with idiopathic pulmonary fibrosis ( $A, C$, and $E$, respectively) and normal lung parenchyma ( $B, D$, and $F$, respectively). Original magnification, $\times 125$.

immunohistochemistry, using previously described criteria, ${ }^{29}$ with slight modifications, from grade 0 to 5 (0, normal lung; 1, minimal fibrous thickening of alveolar or bronchiolar walls; 2, moderate thickening of walls with no obvious damage to lung architecture; 3, increased fibrosis with definite damage to lung structure and formation of fibrous bands or small fibrous masses; 4, severe distortion of structure and large fibrous areas, "honeycomb lung" being placed in this category; 5, total fibrous obliteration throughout the field). The two observers agreed with each other regarding each of the grades.

\section{Analysis of apoptosis in lung tissues}

Apoptosis was detected by the TUNEL method using the DeadEnd colorimetric apoptosis detection system (Promega, Michigan, USA), as described previously. ${ }^{27}$ For each section, the number of positive cells was counted in 20 randomly selected fields at $\times 200$ magnification. The fibrotic grade for each field was also analysed at the same time.

\section{Statistics}

The optical density of western blot analysis and the TUNEL assay, and the number of positive signals for immunohistochemistry, were analysed by the Kruskal Wallis test followed by the Mann-Whitney's U test. Statistics were analysed using Abacus Concepts Stat view 5 package. A $p$ value of less than 0.05 was considered significant.

\section{RESULTS}

\section{Western blot analysis for lung homogenates}

Figure 1 shows representative results of western blot analysis for p53, phosphorylated p53 (at Ser15), and Mdm2 in lung homogenates. These proteins were upregulated in IPF and NSIP compared with the controls. Figure 2 shows the quantitative results of western blot analysis. The expression of p53, phosphorylated p53 (at Ser15), and the Mdm2 protein was significantly higher in IPF $(\mathrm{n}=8)$ and $\operatorname{NSIP}(\mathrm{n}=5)$ than in the controls $(\mathrm{n}=8)$.

\section{Immunohistochemistry and TUNEL staining}

Figures 3 and 4 show that the p53, phosphorylated p53 (at Ser15), Mdm2, Bax, and p2l proteins were detected in a few bronchiolar and alveolar epithelial cells and in a few alveolar macrophages in normal lung parenchyma. In IPF and NSIP, the p53, phosphorylated p53 (at Ser15), and p21 proteins were predominantly detected in the nuclei of bronchiolar and alveolar epithelial cells. Mdm2 protein was detected in the nuclei and cytoplasm of epithelial cells and macrophages and Bax protein was detected in the cytoplasm of epithelial cells.

Figure 5 shows that the number of cells positive for the p53, phosphorylated p53 (at Ser15), Mdm2, Bax, and p2l proteins in bronchiolar and alveolar epithelial cells was significantly higher in IPF and NSIP than in the controls. The number of positive signals for these proteins was significantly higher in IPF than in NSIP, with the exception of the p2l protein. The number of TUNEL positive cells was significantly 

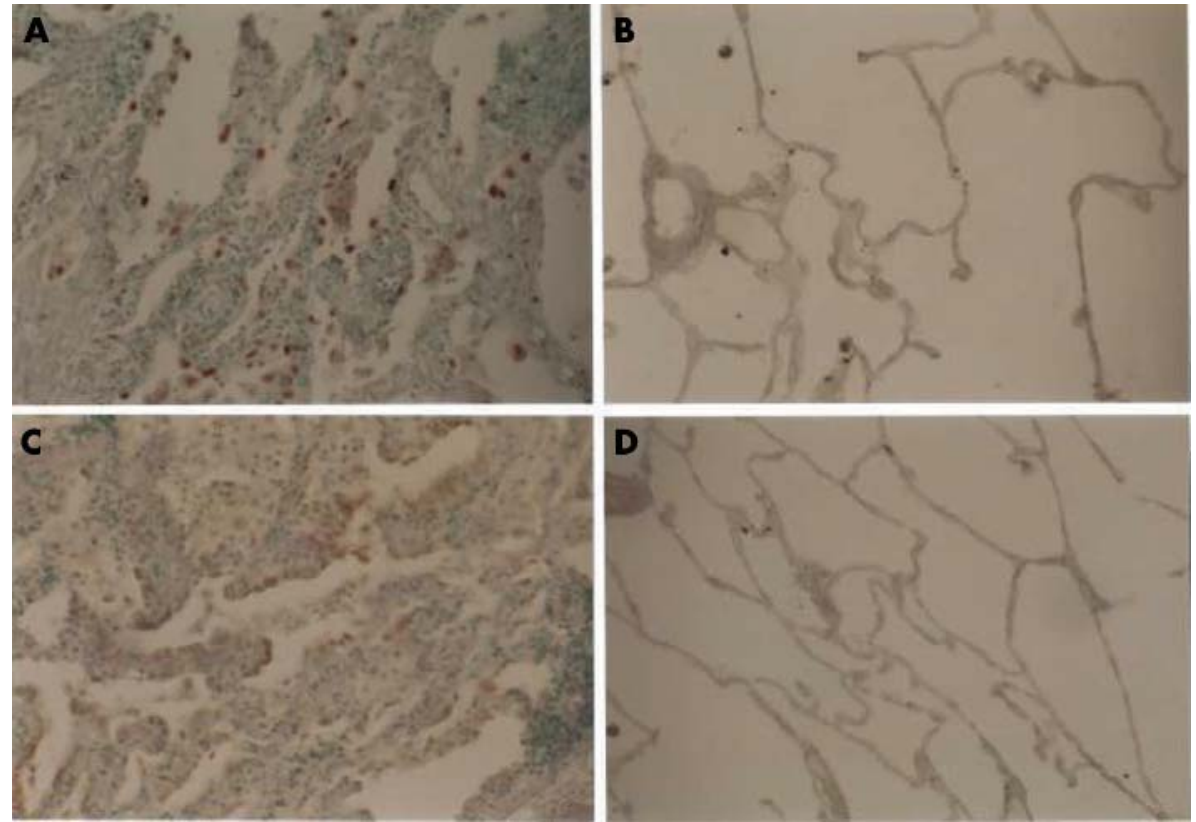

Figure 4 Representative results of immunohistochemical analysis for the 21 and Bax proteins in lung tissue from patients with non-specific interstitial pneumonia ( $A$ and $C$, respectively) and normal lung parenchyma ( $B$ and $D$, respectively). Original magnification, $\times 125$.

higher in IPF and NSIP than in the controls, and was also higher in IPF compared with NSIP, consistent with the findings of our previous study. ${ }^{27}$

Figure 6 shows that positive staining for p53, phosphorylated p53 (at Ser15), Mdm2, and Bax proteins in epithelial cells of IPF and NSIP increased with the progression of pulmonary fibrosis and peaked in moderately fibrotic areas.
Expression of the p2l protein increased with the progression of pulmonary fibrosis and peaked in severely fibrotic areas. Positive staining in the TUNEL assay increased with the progression of pulmonary fibrosis and peaked in moderately fibrotic areas, in the same manner as staining for the p53, phosphorylated p53 (at Ser15), Mdm2, and Bax proteins.

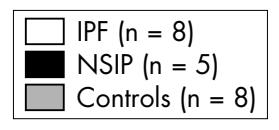

A

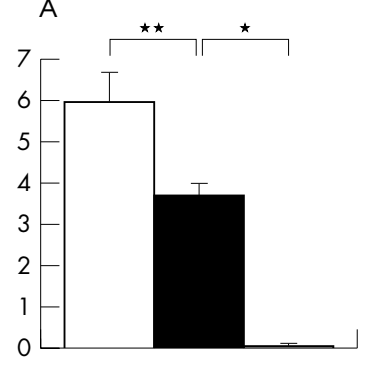

D

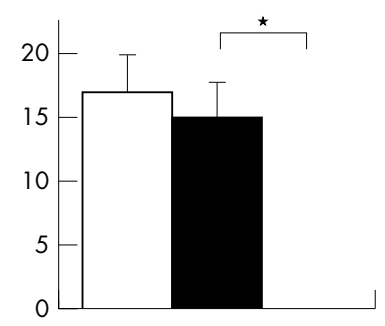

B

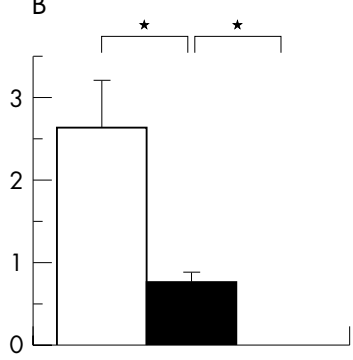

E

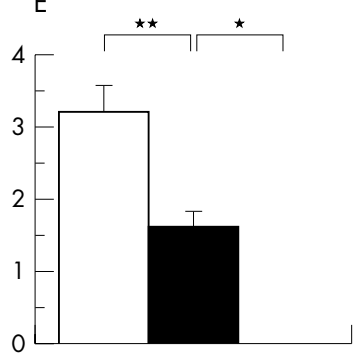

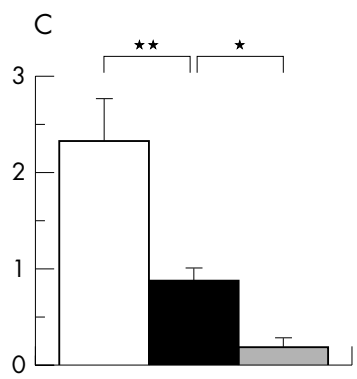

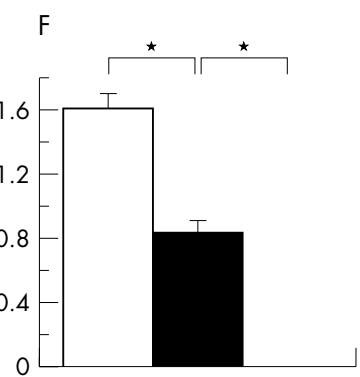

Figure 5 Quantitative results of immunohistochemistry for (A) p53, (B) phosphorylated p53 (at Ser 15), (C) Mdm2, (D) p21, and (E) Bax proteins, in addition to (F) TUNEL staining in lung tissue from patients with idiopathic pulmonary fibrosis (IPF; $\mathrm{n}=8$ ), non-specific interstitial pneumonia (NSIP; $n=5)$, and controls $(n=8)$. The y axis shows the number of positively stained epithelial cells as a percentage of total epithelial cells. ${ }^{*} p<0.01$, ${ }^{* *} \mathrm{p}<0.05$. 
A

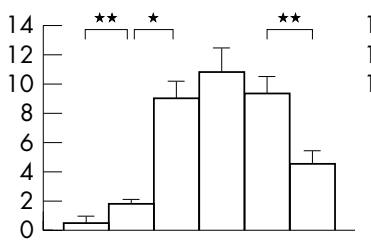

C

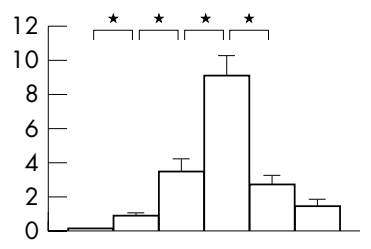

$E$

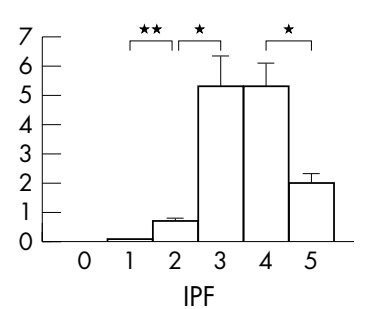

Grade of fibrosis
B

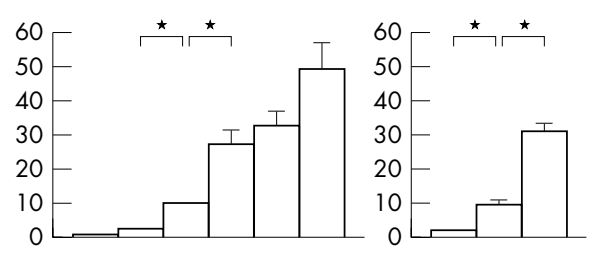

$\mathrm{D}$

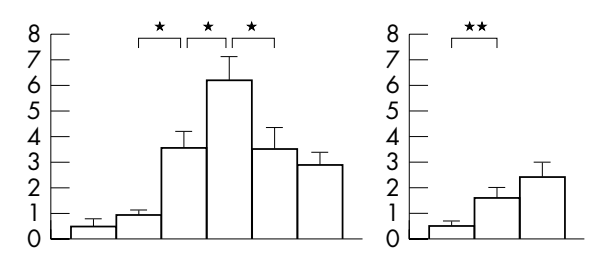

$\mathrm{F}$

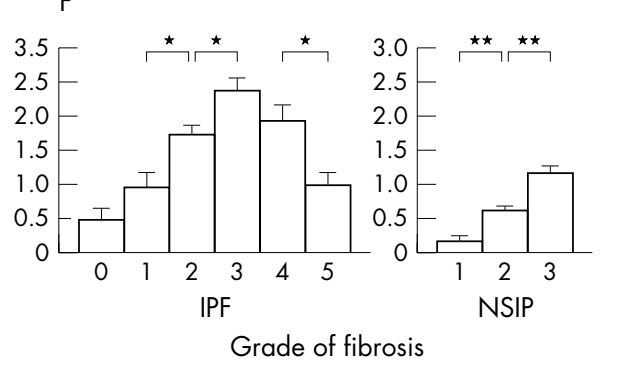

Figure 6 Association between the number of epithelial cells as a percentage of total epithelial cells positively stained (y axis) for (A) p53, (B) p21, (C) phosphorylated p53 (at Ser15), (D) Bax, (E) Mdm2, and (F) TUNEL staining and the grade of pulmonary fibrosis in idiopathic pulmonary fibrosis (IPF) and non-specific interstitial pneumonia (NSIP). ${ }^{*} p<0.01$, ${ }^{* *} p<0.05$ ).

\section{Conjugation with $\mathrm{Mdm} 2$ and ubiquitination of p53}

Figure 7 shows representative results of the association of p53 with Mdm2 and ubiquitination of p53. Immunoblots for immunoprecipitated p53 show that p53 was associated with Mdm2, and that ubiquitinated p53 was found as multiple bands. These results were consistent in repeated experiments. Figure 8 shows the quantitative results of the association of p53 with Mdm2 and ubiquitination of p53. Lower amounts of p53 protein associated with Mdm2 were found in IPF than in NSIP and the controls, and concentrations in NSIP were significantly higher than in the controls (fig 8A). Ubiquitinated p53 was increased in IPF and NSIP compared with the controls (fig 8B).

\section{DISCUSSION}

Upregulation of the p53 protein either induces Gl arrest to allow time for the repair of damaged DNA, or induces apoptosis through transactivation of Bax, DR5, or Fas, or by

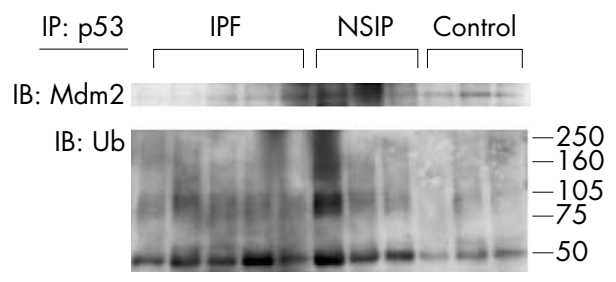

Figure 7 Representative results of the association of $\mathrm{p} 53$ with $\mathrm{Mdm} 2$ and ubiquitination of p53. p53 conjugated with Mdm2 was immunoprecipitated with anti-p53 antibody and analysed by western blot with anti-Mdm2 antibody (upper panel). The molecular weight of the bands is 90 kDa. p53 conjugated with ubiquitin was immunoprecipitated with anti-p53 antibody and then analysed by western blotting with antiubiquitin antibody (lower panel).

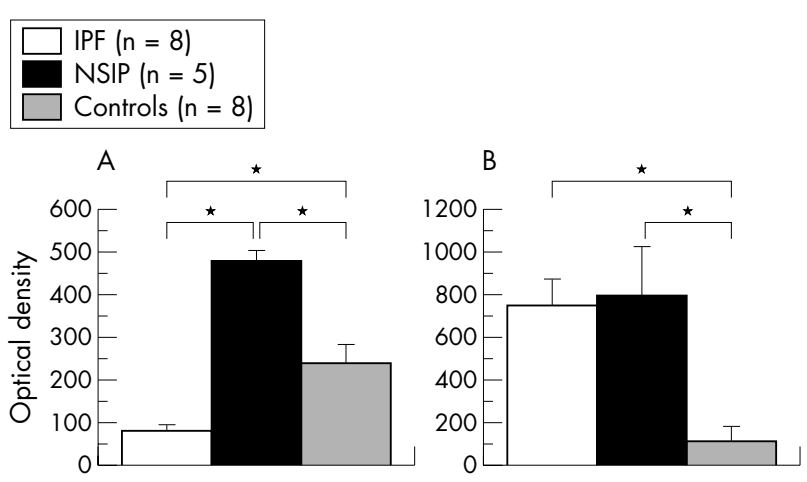

Figure 8 Quantitative results of western blot analysis for the association of (A) p53 with Mdm2 and (B) ubiquitination of p53 in lung tissues from patients with idiopathic pulmonary fibrosis (IPF; $n=8$ ), nonspecific interstitial pneumonia (NSIP; $n=5)$, and controls ( $n=8$ ). * $p<0.05$

transrepressing the expression of Bcl-2. ${ }^{19} 3031$ Apoptotic cells are not specifically detected by the TUNEL assay, which also detects DNA repair, DNA replication, and RNA synthesis. ${ }^{32}$ However, we previously showed that TUNEL positive cells were not the same cells as those that were positive for DNA proliferation or the repair markers, proliferating cell nuclear antigen, Ki-67, and SC-35. ${ }^{27}$ Furthermore, apoptotic epithelial cells were detected in IPF and NSIP by electron microscopy. ${ }^{27}{ }^{33}$ In our study, p53, phosphorylated p53, and Bax were all upregulated in lung epithelial cells from patients with IPF and NSIP compared with normal lung parenchyma. The upregulation of these three factors was similar in magnitude, and was associated with the increase in TUNEL positive cells. These results suggest that p53 accumulation is 
associated with epithelial cell damage and apoptosis in IPF and NSIP.

Several findings have suggested that p21 may be a key regulator of DNA replication and repair following lung injury. ${ }^{22-25}$ Evidence also suggests that p53 induces proapoptotic and antiapoptotic gene expression, and that the balance between proapoptotic genes, such as Bax, and anti-apoptotic genes, such as p2l, is essential for protecting epithelial cells and for promoting the repair of epithelial cell injury. Downstream mediators of p53, such as Bax and p21, are more crucial than p53 itself with regard to sensitivity to hyperoxic lung injury. ${ }^{23} 35$ In our study, the expression of Bax, p53, and phosphorylated p53 in epithelial cells was significantly upregulated in IPF compared with NSIP, and the number of TUNEL positive cells was also increased. However, p21 expression was upregulated in NSIP to the same level as that seen in IPF. These results suggest that DNA damage in the epithelial cells of NSIP is not as severe as in the epithelial cells of IPF, and also suggest that the repair process is predominant over the apoptotic process in NSIP. Because the increase in p2l expression had peaked in severely fibrotic lesions in IPF, and the number of TUNEL positive cells had peaked in moderately fibrotic lesions, but decreased in severely fibrotic lesions in IPF, p2 1 may be a target molecule, in that it inhibits apoptosis if it is strongly expressed at the early fibrosing stage. ${ }^{37}$

Mdm2 plays a central role in regulating p53 expression and in repressing the apoptotic function of $\mathrm{p} 53$. The interaction between $\mathrm{p} 53$ and $\mathrm{Mdm} 2$ appears to determine the degree of expression of the p53 protein, and also appears to be associated with cell fate in lung epithelial cells. In unstressed cells, Mdm2 binds and ubiquitinates p53, causing p53 to exit the nucleus. After DNA damage, phosphorylation of human p53 at Ser 15 occurs, and this leads to reduced interaction of p53 with its negative regulator, Mdm2. ${ }^{15}{ }^{16}$ In nitric oxide stressed cells, phosphorylated p53 at Ser 15 still binds with $\mathrm{Mdm} 2$ and the polyubiquitination of p53 remains intact until functional p53 is no longer needed. ${ }^{38}$ Alveolar epithelial cell injury in IPF is probably mediated, at least in part, by nitric oxide and reactive oxygen species generated at the alveolar epithelial surface. In our study, phosphorylated p53 at Ser 15 was upregulated to the same degree as total p53 in IPF and NSIP. The amount of phosphorylated p53 in epithelial cells was significantly higher in IPF than in NSIP and the controls, and the amount of p53 conjugated with Mdm2 was lower in IPF than in NSIP and the controls. The phosphorylation of p53 in IPF may enable it to escape from Mdm2 binding and degradation, resulting in increased p53 concentrations.

"Our results suggest that DNA damage in the epithelial cells of non-specific interstitial pneumonia (NSIP) is not as severe as in the epithelial cells of idiopathic pulmonary fibrosis, and also suggest that the repair process is predominant over the apoptotic process in NSIP"

Ubiquitination of p53 was found to occur to a similar degree in NSIP and IPF, although conjugation with Mdm2 was much higher in NSIP, suggesting that Mdm2 independent mechanisms for the ubiquitination of $\mathrm{p} 53$ are present in IPF. We previously showed that c-jun N-terminal kinase (JNK) was expressed and activated in epithelial cells in IPF. ${ }^{39}$ JNK has been shown to bind $\mathrm{p} 53$ and to promote ubiquitination and degradation of p53 independent of Mdm2. ${ }^{40}$ In addition, Pirh2, a p53 inducible gene that encodes a RING-H2 protein, has been shown to interact with p53 and promote ubiquitination of p53 independently of Mdm2. ${ }^{41}$ Mdm2, JNK, and Pirh2 may have distinct
Take home messages

- The expression of $\mathrm{p} 53$, phosphorylated $\mathrm{p} 53, \mathrm{Mdm} 2$, p21, and Bax-signalling molecules associated with p53 mediated apoptosis - was upregulated in epithelial cells from patients with idiopathic pulmonary fibrosis (IPF) and non-specific interstitial pneumonia (NSIP) compared with normal lung parenchyma

- Except for p21, there was a significant increase in the expression of these factors in IPF compared with NSIP

- $\mathrm{p} 53-\mathrm{Mdm} 2$ conjugates were decreased in IPF compared with NSIP and controls, and ubiquitinated p53 was increased in IPF and NSIP compared with controls

- Thus, signalling molecules associated with p53 mediated apoptosis may participate in epithelial cell apoptosis, and the attenuation of p53-Mdm2 conjugation and of p53 degradation may be involved in the epithelial cell apoptosis seen in IPF

- Augmented epithelial apoptosis in IPF may lead to the poor prognosis compared with NSIP

mechanisms to regulate the p53 response, and may have important roles in the response to various stresses.

p53 protein concentrations in epithelial cells in IPF were higher than in NSIP, although ubiquitination of p53 occurs to a similar degree in both diseases. These results suggest that the degradation pathway of ubiquitinated p53 may be impaired. Apoptosis associated molecules, such as p53 and bcl-2 family proteins such as Bax, are degraded by proteasomes. Recently, caspases have been shown to cleave specific subunits of the 19S regulatory complex of proteasomes, the role of which is to recognise polyubiquitinated substrates. ${ }^{42}$ We previously demonstrated that caspases 3, 8, and 9 were upregulated and activated in epithelial cells in IPF. ${ }^{27}$ Therefore, the activation of these caspases may inhibit proteasome function and amplify the apoptotic pathway through further accumulation of proapoptotic molecules such as p53 and Bax in IPF.

In conclusion, the expression of proapoptotic proteins and the number of TUNEL positive cells were significantly increased in IPF and NSIP epithelial cells compared with controls. In addition, the expression of proapoptotic proteins and the number of TUNEL positive cells were increased in IPF epithelial cells compared with NSIP, whereas expression of the antiapoptotic protein $\mathrm{p} 2 \mathrm{l}$ was similar in IPF and NSIP epithelial cells. There was a significant decrease in the amount of p53 conjugated with Mdm2 in IPF compared with NSIP and controls, perhaps as a result of the inhibition of binding to $\mathrm{Mdm} 2$ through the phosphorylation of p53 at Ser15 in IPF. Although the association of p53 with Mdm2 was decreased in IPF, ubiquitinated p53 was increased in IPF and NSIP compared with the controls. Caspase activation may inhibit proteasomal recognition and degradation of ubiquitinated p53 through the cleavage of proteasome subunits in IPF. Regulation of $\mathrm{p} 53$ responses by posttranslational modifications including phosphorylation, ubiquitination, and degradation associated with Mdm2 may play an important role in the stress responses of epithelial cells in IPF, and may also be associated with the difference in the prognosis of patients with IPF and NSIP.

\section{ACKNOWLEDGEMENTS}

This work was supported by a Grant-in-Aid for Scientific Research (15590815) from the Ministry of Education, Science and Culture of Japan. 
Authors' affiliations

N Nakashima, K Kuwano, T Maeyama, N Hagimoto, M Yoshimi, N Hamada, M Yamada, Y Nakanishi, Research Institute for Diseases of the Chest, Graduate School of Medical Sciences, Kyushu University, 31-1, Maidashi, Higashi-ku, Fukuoka 812-8582, Japan

\section{REFERENCES}

1 Hershko A, Ciechanover A, Varshavsky A. The ubiquitin system. Nat Med 2000;6: 1073-81

2 Hershko $A$, Ciechanover $A$. The ubiquitin system for protein degradation. Annu Rev Biochem 1992;61:761-807.

3 Ko LJ, Prives C. p53: puzzle and paradigm. Genes Dev 1996; 10:1054-72.

4 Kastan MB, Onyekwere O, Sidransky D, et al. Participation of p53 protein in the cellular response to DNA damage. Cancer Res 1991;51:6304-11.

5 Hubbert NL, Sedman SA, Schiller JT. Human papillomavirus type 16 E6 increases the degradation rate of p53 in human keratinocytes. J Virol 1992;66:6237-41.

6 Maki CG, Howley PM. Ubiquitination of $\mathrm{p} 53$ and $\mathrm{p} 21$ is differentially affected by ionizing and UV radiation. Mol Cell Biol 1997:17:355-63.

7 Maltzman W, Czyzyk L. UV irradiation stimulates levels of p53 cellular tumor antigen in nontransformed mouse cells. Mol Cell Biol 1984;4:1689-94.

8 Honda R, Tanaka H, Yasuda H. Oncoprotein MDM2 is a ubiquitin ligase E3 for tumor suppressor p53. FEBS Lett 1997;420:25-7.

9 Yu ZK, Geyer RK, Maki CG. MDM2-dependent ubiquitination of nuclear and cytoplasmic P53. Oncogene 2000;19:5892-7.

10 Haupt Y, Maya R, Kazaz A, et al. Mdm2 promotes the rapid degradation of p53. Nature 1997;387:296-9.

11 Kubbutat MH, Jones SN, Vousden KH. Regulation of p53 stability by Mdm2. Nature 1997:387:299-303.

12 Yap DB, Hsieh JK, Lu X. Mdm2 inhibits the apoptotic function of p53 mainly by targeting it for degradation. J Biol Chem 2000;275:37296-302.

13 Momand J, Zambetti GP, Olson DC, et al. The mdm-2 oncogene product forms a complex with the $\mathrm{p} 53$ protein and inhibits $\mathrm{p} 53$-mediated transactivation. Cell 1992;69:1237-45.

14 Oliner JD, Pietenpol JA, Thiagalingam S, et al. Oncoprotein MDM2 conceals the activation domain of tumour suppressor p53. Nature 1993;362:857-60.

15 Prives C. Signaling to p53. Breaking the MDM2-p53 circuit. Cell 1998:95:5-8

16 Shieh SY, Ikeda M, Taya Y, et al. DNA damage-induced phosphorylation of p53 alleviates inhibition by MDM2. Cell 1997;91:325-34.

17 Zhang Y, Xiong Y. A p53 amino-terminal nuclear export signal inhibited by DNA damage-induced phosphorylation. Science 2001;292:1910-15.

18 Johnstone RW, Ruefli AA, Lowe SW. Apoptosis: a link between cancer genetics and chemotherapy. Cell 2002;108:153-64.

19 Miyashita T, Reed JC. Tumor suppressor p53 is a direct transcriptional activator of the human bax gene. Cell 1995;80:293-9.

20 Guinee D Jr, Fleming M, Hayashi T, et al. Association of p53 and WAF1 expression with apoptosis in diffuse alveolar damage. Am J Pathol 1996;149:531-8.

21 Guinee D Jr, Brambilla E, Fleming M, et al. The potential role of BAX and BCL2 expression in diffuse alveolar damage. Am J Pathol 1997;151:999-1007.

22 El-Deiry WS, Harper JW, O'Connor PM, et al. WAF1/CIP1 is induced in p53mediated G1 arrest and apoptosis. Cancer Res 1994;54:1169-74.
23 O'Reilly MA, Staversky RJ, Watkins RH, et al. The cyclin-dependent kinase inhibitor 21 protects the lung from oxidative stress. Am J Respir Cell Mol Biol $2001 ; 24: 703-10$

24 Bissonnette N, Hunting DJ. p21-induced cycle arrest in G1 protects cells from apoptosis induced by UV-irradiation or RNA polymerase II blockage. Oncogene 1998; 16:3461-9.

25 Lu Y, Yamagishi N, Yagi T, et al. Mutated p21(WAF1/CIP1/SDI1) lacking CDK-inhibitory activity fails to prevent apoptosis in human colorectal carcinoma cells. Oncogene 1998;16:705-712.

26 Kuwano K, Kunitake R, Kawasaki M, et al. p21Wafl/Cipl/Sdi1 and p53 expression in association with DNA strand breaks in idiopathic pulmonary fibrosis. Am J Respir Crit Care Med 1996;15:477-83.

27 Kuwano K, Hagimoto N, Maeyama T, et al. Mitochondria-mediated apoptosis of lung epithelial cells in idiopathic interstitial pneumonias. Lab Invest 2002;82:1695-706.

28 American Thoracic Society. Idiopathic pulmonary fibrosis: diagnosis and treatment. International consensus statement. Am J Respir Crit Care Med 2000;16:646-64.

29 Ashcroft T, Simpson JM, Timbrell V. Simple method of estimating severity of pulmonary fibrosis on a numerical scale. J Clin Pathol 1988;41:467-70.

30 Wu GS, Burns TF, McDonald ER, et al. KILLER/DR5 is a DNA damageinducible p53-regulated death receptor gene. Nat Genet 1997;17:141-3.

31 Muller M, Wilder S, Bannasch D, et al. p53 activates the CD95 (APO-1/Fas) gene in response to DNA damage by anticancer drugs. J Exp Med 1998; 188:2033-45.

32 Kockx MM, Muhring J, Knaapen MW, et al. RNA synthesis and splicing interferes with DNA in situ end labeling techniques used to detect apoptosis. Am J Pathol 1998; 152:885-8.

33 Barbas-Filho JV, Ferreira MA, Sesso A, et al. Evidence of type II pneumocyte apoptosis in the pathogenesis of idiopathic pulmonary fibrosis (IFP)/usual interstitial pneumonia (UIP). J Clin Pathol 2001;54:132-8.

34 Uhal BD, Joshi I, Hughes WF, et al. Alveolar epithelial cell death adjacent to underlying myofibroblasts in advanced fibrotic human lung. Am J Physiol 1998;275:L1 192-9.

35 Barazzone C, Horowitz S, Donati YR, et al. Oxygen toxicity in mouse lung: pathways to cell death. Am J Respir Cell Mol Biol 1998;19:573-81.

36 Budinger GR, Tso M, McClintock DS, et al. Hyperoxia-induced apoptosis does not require mitochondrial reactive oxygen species and is regulated by $\mathrm{Bcl}-2$ proteins. J Biol Chem 2002;277:15654-60

37 Inoshima I, Kuwano K, Hamada N, et al. Induction of CDK inhibitor p21 gene as a new therapeutic strategy against pulmonary fibrosis. Am J Physiol Lung Cell Mol Physiol 2004;286:L727-33.

38 Schneiderhan N, Budde A, Zhang Y, et al. Nitric oxide induces phosphorylation of p53 and impairs nuclear export. Oncogene 2003;22:2857-68.

39 Yoshida K, Kuwano K, Hagimoto N, et al. MAP kinase activation and apoptosis in lung tissues from patients with idiopathic pulmonary fibrosis. J Pathol 2002; 198:388-96.

40 Fuchs SY, Adler V, Buschmann T, et al. JNK targets p53 ubiquitination and degradation in nonstressed cells. Genes Dev 1998;12:2658-63.

41 Leng RP, Lin Y, Ma W, et al. Pirh2, a p53-induced ubiquitin-protein ligase, promotes p53 degradation. Cell 2003;112:779-91.

42 Sun XM, Butterworth $M$, MacFarlane $M$, et al. Caspase activation inhibits proteasome function during apoptosis. Mol Cell 2004;14:81-93. 